\title{
EXISTENCE OF TANGENT LINES TO CARNOT-CARATHÉODORY GEODESICS
}

\author{
ROBERTO MONTI, ALESSANDRO PIGATI, AND DAVIDE VITTONE
}

\begin{abstract}
We show that length minimizing curves in Carnot-Carathéodory spaces possess at any point at least one tangent curve (i.e., a blow-up in the nilpotent approximation) equal to a straight horizontal line. This is the first regularity result for length minimizers that holds with no assumption on either the space (e.g., its rank, step, or analyticity) or the curve, and it is novel even in the setting of Carnot groups.
\end{abstract}

\section{INTRODUCTION}

Let $M$ be a connected $n$-dimensional $C^{\infty}$-smooth manifold and $\mathscr{X}=\left\{X_{1}, \ldots, X_{r}\right\}$, $r \geq 2$, a system of linearly independent $C^{\infty}$-smooth vector fields on $M$ satisfying the Hörmander condition. We call the pair $(M, \mathscr{X})$ a Carnot-Carathéodory $(C C)$ structure. Given an interval $I \subseteq \mathbb{R}$, a Lipschitz curve $\gamma: I \rightarrow M$ is said to be horizontal if there exist functions $h_{1}, \ldots, h_{r} \in L^{\infty}(I)$ such that for a.e. $t \in I$ we have

$$
\dot{\gamma}(t)=\sum_{i=1}^{r} h_{i}(t) X_{i}(\gamma(t))
$$

Letting $|h|:=\left(h_{1}^{2}+\ldots+h_{r}^{2}\right)^{1 / 2}$, the length of $\gamma$ is then defined as

$$
L(\gamma):=\int_{I}|h(t)| d t
$$

We will usually assume that curves are parameterized by arclength, i.e., $|h(t)|=1$ for a.e. $t$, so that $\mathscr{L}^{1}(I)=L(\gamma)$.

Since $M$ is connected, by Chow-Rashevsky theorem for any pair of points $x, y \in M$ there exists a horizontal curve joining $x$ to $y$. We can therefore define a distance function $d: M \times M \rightarrow[0, \infty)$ letting

$$
d(x, y):=\inf \{L(\gamma) \mid \gamma:[0, T] \rightarrow M \text { horizontal with } \gamma(0)=x \text { and } \gamma(T)=y\} .
$$

2010 Mathematics Subject Classification. 53C17, 49K30, 28A75.

Key words and phrases. Geodesics, regularity of length minimizers, Carnot-Carathéodory spaces, sub-Riemannian geometry, Carnot groups.

R. M. and D. V. are supported by MIUR (Italy) and University of Padova. D. V. is supported by University of Padova Project Networking and INdAM-GNAMPA Project 2017 "Campi vettoriali, superfici e perimetri in geometrie singolari". 
The resulting metric space $(M, d)$ is a Carnot-Carathéodory space. Typical examples of Carnot-Carathéodory spaces are given by sub-Riemannian manifolds $(M, \mathscr{D}, g)$, where $\mathscr{D} \subset T M$ is a completely non-integrable distribution and $g$ is a smooth metric on $\mathscr{D}$.

If the closure of any ball in $(M, d)$ is compact, then the infimum in (1.2) is a minimum, i.e., any pair of points can be connected by a length-minimizing curve. A horizontal curve $\gamma:[0, T] \rightarrow M$ is a length minimizer if $L(\gamma)=d(\gamma(0), \gamma(T))$. In Carnot-Carathéodory spaces (or even in the model case of Carnot groups) it is not known whether constant-speed length minimizers are $C^{\infty}$-smooth, or even $C^{1}$ smooth. The main obstacle is the presence of abnormal length minimizers, which are not captured by the natural Hamiltonian framework, see e.g. [17, Section 1.5]. In [16], Montgomery gave the first example of such a length minimizer. Contrary to the Riemannian case, stationarity conditions do not guarantee any smoothness of the curve: in [12] it is proved that no further regularity beyond the Lipschitz one can be obtained for abnormal extremals from the Pontryagin Maximum Principle and the Goh condition (which is a second-order necessary condition, see e.g. [2, Chapter 20]).

However, some partial regularity results are known. If the step is at most 2 (i.e., for any $x$ the tangent space $T_{x} M$ is spanned by the $r+\left(\begin{array}{l}r \\ 2\end{array}\right)$ vectors $\left.X_{i}(x),\left[X_{i}, X_{j}\right](x)\right)$, then all constant-speed length minimizers are smooth. In the context of Carnot groups, the regularity problem was recently solved also when the step is at most 3 by Le Donne, Leonardi, Monti and Vittone in [11]. In [22] Sussmann proved that, in presence of analytic data (and in particular in Carnot groups), all length minimizers are analytic on a dense open set of times, although it is not known whether this set has full measure. Building on ideas contained in [14, 13, Hakavuori and Le Donne recently proved in [7] that length minimizers cannot have corner-type singularities. Other partial regularity results are contained in [18]. We also refer to [1, 19, 21, 23] for surveys about the known results on the problem.

It is well-known that, at any point $x \in M$, the space $(M, \mathscr{X})$ has a nilpotent approximation $\left(M^{\infty}, \mathscr{X}^{\infty}\right)$, which is itself a Carnot-Carathéodory structure. The corresponding metric space is obtained as a pointed Gromov-Hausdorff limit of metric spaces. An elementary construction of $\left(M^{\infty}, \mathscr{X}^{\infty}\right)$ is detailed in [20].

When $t \in(-T, T)$ is fixed and we perform this construction for $x=\gamma(t)$, we denote by $\operatorname{Tan}(\gamma ; t)$ the set containing all possible curves in $M^{\infty}$ that arise as limits of $\gamma$ in the local uniform topology. The tangent cone $\operatorname{Tan}(\gamma ; t)$ was introduced in [20], where it was also proved that its elements are length minimizing horizontal curves in $M^{\infty}$ parametrized by arclength. We call horizontal line any horizontal curve in $\left(M^{\infty}, \mathscr{X}^{\infty}\right)$ passing through the base point in $M^{\infty}$ and with constant controls.

The following theorem is the main result of the paper. 
Theorem 1.1. Let $\gamma:[-T, T] \rightarrow M$ be a length minimizer parametrized by arclength in a Carnot-Carathéodory space $(M, d)$. Then, for any $t \in(-T, T)$, the tangent cone $\operatorname{Tan}(\gamma ; t)$ contains a horizontal line.

Theorem 1.1 has an analytic reformulation, stated solely in terms of the control $h$, which is independent of the notion of nilpotent approximation: see Remark 4.3, A version of Theorem 1.1 holds for the extremal points $t=0$ and $t=T$ of a length minimizer $\gamma:[0, T] \rightarrow M$. In this case, the tangent cone contains a horizontal half-line; see Theorem 4.2. These results imply and improve the ones contained in [14, 13, 7]: while in these papers the existence of (linearly independent) left and right derivatives is assumed in order to construct a shorter competitor, Theorem 1.1 provides a mild form of pointwise differentiability which automatically rules out corner-type singularities.

Theorem 1.1 is deduced from a similar result for the case when $M=G$ is a Carnot group of rank $r \geq 2$ and $\mathscr{X}=\left\{X_{1}, \ldots, X_{r}\right\}$ is a system of left-invariant vector fields forming a basis of the first layer of its Lie algebra $\mathfrak{g}$. As explained in the proof of Theorem 1.1, the reduction to this case is made possible by the results proved in [20].

The proof in the case of a Carnot group, in turn, is a consequence of Theorem 1.2 below. Let $\mathfrak{g}=\mathfrak{g}_{1} \oplus \cdots \oplus \mathfrak{g}_{s}$ be the stratification of $\mathfrak{g}$ and let $\langle\cdot, \cdot\rangle$ be the scalar product on $\mathfrak{g}_{1}$ making $X_{1}, \ldots, X_{r}$ orthonormal. The integer $s \geq 2$ is the step of the group and $r=\operatorname{dim} \mathfrak{g}_{1}$ its rank. We denote by $S^{r-1}=\left\{v \in \mathfrak{g}_{1}:\langle v, v\rangle=1\right\}$ the unit sphere in $\mathfrak{g}_{1}$. We define the excess of a horizontal curve $\gamma:[-T, T] \rightarrow G$ over a Borel set $B \subseteq[-T, T]$ with positive measure as

$$
\operatorname{Exc}(\gamma ; B):=\inf _{v \in S^{r-1}}\left(f_{B}\langle v, \dot{\gamma}(t)\rangle^{2} d t\right)^{1 / 2} .
$$

The excess $\operatorname{Exc}(\gamma ; B)$ measures how far $\dot{\gamma}_{\mid B}$ is from being contained in a single hyperplane of $\mathfrak{g}_{1}$, see Remark 2.3. For length-minimizing curves, the excess is infinitesimal at suitably small scales, as stated in our second main result.

Theorem 1.2. Let $G$ be a Carnot group and let $\gamma:[-T, T] \rightarrow G, T>0$, be a length-minimizing curve parametrized by arclength. Then there exists an infinitesimal sequence $\eta_{i} \downarrow 0$ such that

$$
\lim _{i \rightarrow \infty} \operatorname{Exc}\left(\gamma ;\left[-\eta_{i}, \eta_{i}\right]\right)=0
$$

Again, this result has a version for extremal points: for a length minimizer $\gamma$ : $[0, T] \rightarrow G$ the excess $\operatorname{Exc}\left(\gamma ;\left[0, \eta_{i}\right]\right)$ is infinitesimal, see Theorem 4.1. When $r=2$, (1.3) implies that there exists $\kappa \in \operatorname{Tan}(\gamma ; 0)$ of the form $\kappa(t)=\exp (t v)$ for some $v \in \mathfrak{g}_{1}$. This proves Theorem 1.1 for $M=G$ with $r=2$. When $r>2$, the situation can be reduced by induction to the case $r=2$ using, again, the outcomes of [20]. 
The introduction of the excess is probably among the main contributions of this paper; the reader familiar with the regularity theory of minimal hypersurfaces in $\mathbb{R}^{n}$ will notice the analogy with the quantity that plays a key role in De Giorgi's approach to that problem, see e.g. [6], and in many subsequent results inspired by his work (we just mention e.g. [3] and [5]). In this sense, Theorems 1.1 and 1.2 constitute a first step towards a regularity theory for length minimizers, whose next stages (height bounds, Lipschitz approximation theorems, reverse Poincaré inequality, harmonic approximation according to the terminology of [15]) could now see their way paved by Theorems 1.1 and 1.2 .

We conclude this introduction by spending a few words about the proof of Theorem 1.2. As detailed in Section 4, it goes by contradiction and uses a cut-and-adjust construction performed in $s$ steps. If we had $\operatorname{Exc}(\gamma ;[-\eta, \eta]) \geq \varepsilon$ for some $\varepsilon>0$ and for all small $\eta>0$, then we could find $t_{1}<\cdots<t_{r}$ such that, roughly speaking, the vectors $\dot{\gamma}\left(t_{1}\right), \ldots, \dot{\gamma}\left(t_{r}\right) \in \mathfrak{g}_{1}$ are linearly independent in a quantitative way, see Lemma 2.7. We could replace the "horizontal projection" $\underline{\gamma}$ of $\gamma$ on the interval $[-\eta, \eta]$ with the line segment joining $\underline{\gamma}(-\eta)$ to $\underline{\gamma}(\eta)$, whose gain of length would be estimated from below in terms of the excess, see Lemma 3.4, and we could lift the resulting "horizontal coordinates" to a horizontal curve in $G$. The end-point of the new curve might be different, but the vectors $\dot{\gamma}\left(t_{1}\right), \ldots, \dot{\gamma}\left(t_{r}\right)$ could then be used to build suitable correction devices restoring the end-point, taking care to keep a positive gain of length. This construction is detailed in Sections 2 , 3 and 4 and it is a refinement of the techniques introduced and developed in [14] and [7]. In particular, Section 3 contains explicit formulas, for the length gain associated with the cut and for the displacement of the final point caused by the application of the correction devices, which make the constructions in [14, 7] more transparent. We believe that these formulas have an independent interest and could possibly be useful for future applications.

Acknowledgements. We thank L. Ambrosio for several discussions and for being an invaluable mentor and friend.

\section{ExCESS, COMPACTNESS OF LENGTH MINIMIZERS AND FIRST CONSEQUENCES}

In this section we prove Lemma 2.7, which provides the correct position for the correction devices introduced in Section 3. We work in the setting of a Carnot group.

Definition 2.1. A Carnot group is a finite dimensional connected, simply connected and nilpotent Lie group $G$ whose Lie algebra $\mathfrak{g}$ is stratified, i.e., there exists a (fixed) decomposition $\mathfrak{g}=\mathfrak{g}_{1} \oplus \cdots \oplus \mathfrak{g}_{s}$ such that $\mathfrak{g}_{j}=\left[\mathfrak{g}_{1}, \mathfrak{g}_{j-1}\right]$ for any $j=2, \ldots, s$ and $\left[\mathfrak{g}, \mathfrak{g}_{s}\right]=\{0\}$. 
We will denote by $n$ the dimension of $\mathfrak{g}$ and by $r$ the dimension of its first layer $\mathfrak{g}_{1}$; we refer to the integers $r, s$ as the rank and step of $\mathfrak{g}$, respectively. We endow $\mathfrak{g}$ with a positive definite scalar product $\langle\cdot, \cdot\rangle$ such that $\mathfrak{g}_{i} \perp \mathfrak{g}_{j}$ whenever $i \neq j$. We also let $|\cdot|:=\langle\cdot, \cdot\rangle^{1 / 2}$. We fix an orthonormal basis $X_{1}, \ldots, X_{n}$ of $\mathfrak{g}$ adapted to the stratification, i.e., such that $\mathfrak{g}_{j}=\operatorname{span}\left\{X_{r_{j-1}+1}, \ldots, X_{r_{j}}\right\}$ for any $j=1, \ldots, s$, where $r_{j}:=\operatorname{dim}\left(\mathfrak{g}_{1}\right)+\cdots+\operatorname{dim}\left(\mathfrak{g}_{j}\right)$ and $r_{0}:=0$.

For $\lambda>0$, the dilations $\delta_{r}: \mathfrak{g} \rightarrow \mathfrak{g}$ defined by

$$
\delta_{\lambda}(X):=\lambda^{j} X, \quad \text { if } X \in \mathfrak{g}_{j},
$$

form a one-parameter group of isomorphisms of $\mathfrak{g}$. Being $\mathfrak{g}$ nilpotent, the exponential map exp $: \mathfrak{g} \rightarrow G$ is a diffeomorphism and, by composition with exp, the dilations on $\mathfrak{g}$ induce a one-parameter family of group isomorphisms, which we still denote by $\delta_{\lambda}: G \rightarrow G$. We recall for future reference the Baker-Campbell-Hausdorff formula: for any $X, Y \in \mathfrak{g}$ we have $\exp (X) \exp (Y)=\exp (P(X, Y))$, where

$$
P(X, Y):=\sum_{p=1}^{s} \frac{(-1)^{p+1}}{p} \sum_{1 \leq k_{i}+\ell_{i} \leq s} \frac{\left[X^{k_{1}}, Y^{\ell_{1}}, \ldots, X^{k_{p}}, Y^{\ell_{p}}\right]}{k_{1} ! \cdots k_{p} ! \ell_{1} ! \cdots \ell_{p} ! \sum_{i}\left(k_{i}+\ell_{i}\right)} .
$$

Here, we use the short notation $\left[Z_{1}, \ldots, Z_{k+1}\right]:=\left(\operatorname{ad} Z_{1}\right) \cdots\left(\operatorname{ad} Z_{k}\right) Z_{k+1}$, with ad $X$ : $\mathfrak{g} \rightarrow \mathfrak{g}$ being the adjoint mapping ad $X(Y):=[X, Y]$.

The group $G$ is endowed with the Carnot-Carathéodory distance $d$ induced by the family $X_{1}, \ldots, X_{r}$, for which one clearly has, for $x, y, z \in G$ and $\lambda>0$,

$$
d(z x, z y)=d(x, y) \quad \text { and } \quad d\left(\delta_{\lambda}(x), \delta_{\lambda}(y)\right)=\lambda d(x, y) .
$$

We will frequently use the homogeneous (pseudo-)norm $\|x\|$ defined in this way: if $x=\exp \left(Y_{1}+\cdots+Y_{s}\right)$ for $Y_{j} \in \mathfrak{g}_{j}$, then

$$
\|x\|:=\sum_{j=1}^{s}\left|Y_{j}\right|^{1 / j}
$$

A well-known consequence of the homogeneity of $\|\cdot\|$ and (2.5) is the fact that $\|\cdot\|$ is equivalent to the distance function from the identity 0 of $G$. In particular there exists $C>0$ such that, for any horizontal curve $\gamma:[-T, T] \rightarrow G$ parametrized by arclength and such that $\gamma(0)=0$, the estimate

$$
\|\gamma(t)\| \leq C|t|, \quad t \in[-T, T]
$$

holds.

We introduce one of the main objects of this paper, the excess of a horizontal curve. Let us denote by $\bar{\pi}: \mathfrak{g} \rightarrow \mathfrak{g}_{1}$ the projection onto the first layer and by $\pi: G \rightarrow \mathfrak{g}_{1}$ the map $\pi:=\pi \circ \exp ^{-1}$. For any curve $\gamma$ in $G$ we use the short notation $\underline{\gamma}:=\pi \circ \gamma$. We also identify $\mathfrak{g}_{1}$ with $\mathbb{R}^{r}$ through the fixed orthonormal basis $X_{1}, \ldots, X_{r}$ and denote 
by $S^{r-1}$ and $G(r-1)$ the set of unit vectors and linear hyperplanes in $\mathfrak{g}_{1}$, respectively. For the rest of this section, $I$ denotes a compact interval of positive length.

Definition 2.2. Given a horizontal curve $\gamma: I \rightarrow G$ and a Borel subset $B \subseteq I$ with $\mathscr{L}^{1}(B)>0$, we define the excess of $\gamma$ on $B$ as

$$
\operatorname{Exc}(\gamma ; B):=\inf _{v \in S^{r-1}}\left(f_{B}\langle v, \dot{\gamma}(t)\rangle^{2} d t\right)^{1 / 2}
$$

Remark 2.3. The excess can be equivalently defined as

$$
\operatorname{Exc}(\gamma ; B):=\inf _{\Pi \in G(r-1)}\left(f_{B}|\dot{\gamma}(t)-\Pi(\underline{\dot{\gamma}}(t))|^{2} d t\right)^{1 / 2},
$$

where we identify the hyperplane $\Pi$ with the orthogonal projection $\mathfrak{g}_{1} \rightarrow \Pi$.

Remark 2.4. Given a horizontal curve $\gamma, g \in G$ and $r>0$, setting $\gamma_{1}(t):=g \gamma(t)$, $\gamma_{2}(t):=\delta_{r}(\gamma(t))$, we have

$$
\operatorname{Exc}\left(\gamma_{1} ; B\right)=\operatorname{Exc}(\gamma ; B) \text { and } \operatorname{Exc}\left(\gamma_{2} ; B\right)=r \operatorname{Exc}(\gamma ; B) .
$$

Moreover, for $\gamma_{3}(t):=\delta_{r}(\gamma(t / r))$ we have $\operatorname{Exc}\left(\gamma_{3} ; r B\right)=\operatorname{Exc}(\gamma ; B)$.

Remark 2.5. The map

$$
S^{r-1} \times L^{2}\left(I, \mathfrak{g}_{1}\right) \ni(v, u) \mapsto\left(f_{B}\langle v, u(t)\rangle^{2} d t\right)^{1 / 2} \in \mathbb{R}
$$

is continuous. As a consequence, the infimum in Definition 2.2 is in fact a minimum and, by the compactness of $S^{r-1}$, we have

$$
\operatorname{Exc}\left(\gamma_{k} ; B\right) \rightarrow \operatorname{Exc}(\gamma ; B)
$$

whenever $\underline{\dot{\gamma}_{k}} \rightarrow \underline{\dot{\gamma}}$ in $L^{2}\left(I, \mathfrak{g}_{1}\right)$.

The following compactness result for length minimizers parametrized by arclength implies a certain uniform - though not explicit - estimate: see Lemma 2.7 below.

Lemma 2.6 (Compactness of minimizers). Let $I$ be a compact interval and let $\gamma_{k}$ : $I \rightarrow G, k \in \mathbb{N}$, be a sequence of length minimizers parametrized by arclength with $\gamma_{k}\left(t_{0}\right)=0$, for a fixed $t_{0} \in I$. Then, there exist a subsequence $\gamma_{k_{p}}$ and a length minimizer $\gamma_{\infty}: I \rightarrow G$, parametrized by arclength and with $\gamma_{\infty}\left(t_{0}\right)=0$, such that $\gamma_{k_{p}} \rightarrow \gamma_{\infty}$ uniformly and $\underline{\dot{\gamma}_{k_{p}}} \rightarrow \underline{\dot{\gamma}_{\infty}}$ in $L^{2}(I)$.

Proof. By homogeneity, it is not restrictive to assume $I=[0,1]$. For any $k$ we have $\gamma_{k}([0,1]) \subseteq \overline{B(0,1)}$, the closed unit ball, which is compact. Since all the curves $\gamma_{k}$ are 1-Lipschitz with respect to the Carnot-Carathéodory distance $d$, we can find a subsequence $\gamma_{k_{p}}$ converging uniformly to some curve $\gamma_{\infty}$. 
Let $u_{p}:=\dot{\gamma}_{k_{p}}$. By $(3.13)$ one has $\left|u_{p}\right|=1$ a.e., so up to selecting a further subsequence we can assume that $u_{p} \rightarrow u_{\infty}$ in $L^{2}\left([0,1], \mathfrak{g}_{1}\right)$. Thus, identifying $G$ with $\mathbb{R}^{n}$ by exponential coordinates and passing to the limit as $p \rightarrow \infty$ in

$$
\gamma_{k_{p}}(t)=\int_{0}^{t}\left(\sum_{i=1}^{r} u_{p, i}(\tau) X_{i}\left(\gamma_{k_{p}}(\tau)\right)\right) d \tau
$$

(which holds again by (3.13) ), we obtain, for any $t \in[0,1]$,

$$
\gamma_{\infty}(t)=\int_{0}^{t}\left(\sum_{i=1}^{r} u_{\infty, i}(\tau) X_{i}\left(\gamma_{\infty}(\tau)\right)\right) d \tau .
$$

This proves that $\gamma_{\infty}$ is horizontal with $\underline{\dot{\gamma}_{\infty}}=u_{\infty}$. Moreover,

$$
\left\|u_{\infty}\right\|_{L^{2}\left([0,1], \mathfrak{g}_{1}\right)} \geq L\left(\gamma_{\infty}\right) \geq d\left(\gamma_{\infty}(0), \gamma_{\infty}(1)\right)=\lim _{p \rightarrow \infty} d\left(\gamma_{k_{p}}(0), \gamma_{k_{p}}(1)\right)=1
$$

We already know that $\left\|u_{\infty}\right\|_{L^{2}\left([0,1], \mathfrak{g}_{1}\right)} \leq 1$ (because $u_{p} \rightarrow u_{\infty}$ and $\left\|u_{p}\right\|_{L^{2}\left([0,1], \mathfrak{g}_{1}\right)}=1$ ), so $\left\|u_{p}\right\|_{L^{2}\left([0,1], \mathfrak{g}_{1}\right)} \rightarrow\left\|u_{\infty}\right\|_{L^{2}\left([0,1], \mathfrak{g}_{1}\right)}$ and, since $L^{2}\left([0,1], \mathfrak{g}_{1}\right)$ is a Hilbert space, this gives $u_{p} \rightarrow u_{\infty}$ in $L^{2}\left([0,1], \mathfrak{g}_{1}\right)$. In particular, $\underline{\dot{\gamma}}_{\infty}(t)$ is for a.e. $t \in[0,1]$ a unit vector in $\mathfrak{g}_{1}$. As all inequalities in (2.7) must be equalities, we obtain $L\left(\gamma_{\infty}\right)=d\left(\gamma_{\infty}(0), \gamma_{\infty}(1)\right)$, i.e., $\gamma_{\infty}$ is a length minimizer parametrized by arclength.

Lemma 2.7. For any $\varepsilon>0$ there exists a constant $c=c(G, \varepsilon)>0$ such that the following holds. For any length minimizer $\gamma: I \rightarrow G$ parametrized by arclength and such that $\operatorname{Exc}(\gamma ; I) \geq \varepsilon$, there exist $r$ subintervals $\left[a_{1}, b_{1}\right], \ldots,\left[a_{r}, b_{r}\right] \subseteq I$, with $a_{i}<b_{i} \leq a_{i+1}$, such that

$$
\left|\operatorname{det}\left(\underline{\gamma}\left(b_{1}\right)-\underline{\gamma}\left(a_{1}\right), \ldots, \underline{\gamma}\left(b_{r}\right)-\underline{\gamma}\left(a_{r}\right)\right)\right| \geq c\left(\mathscr{L}^{1}(I)\right)^{r} .
$$

The determinant is defined by means of the identification of $\mathfrak{g}_{1}$ with $\mathbb{R}^{r}$ via the basis $X_{1}, \ldots, X_{r}$.

Proof. By Remark 2.4 we can assume that $I=[0,1]$ and that $\gamma(0)=0$. By contradiction, assume there exist length minimizers $\gamma_{k}:[0,1] \rightarrow G$ parametrized by arclength, with $\gamma_{k}(0)=0$ and $\operatorname{Exc}\left(\gamma_{k} ;[0,1]\right) \geq \varepsilon$, such that

$$
\left|\operatorname{det}\left(\underline{\gamma_{k}}\left(b_{1}\right)-\underline{\gamma_{k}}\left(a_{1}\right), \ldots, \underline{\gamma_{k}}\left(b_{r}\right)-\underline{\gamma_{k}}\left(a_{r}\right)\right)\right| \leq 2^{-k},
$$

for any $0 \leq a_{1}<b_{1} \leq \cdots \leq a_{r}<b_{r} \leq 1$. By Lemma 2.6, there exists a subsequence $\left(\gamma_{k_{p}}\right)_{p \in \mathbb{N}}$ such that $\gamma_{k_{p}} \rightarrow \gamma_{\infty}$ uniformly and $\dot{\gamma}_{k_{p}} \rightarrow \underline{\dot{\gamma}_{\infty}}$ in $L^{2}([0,1])$ for some length minimizer $\gamma_{\infty}$ parametrized by arclength. Passing to the limit as $p \rightarrow \infty$ in (2.9) we deduce that

$$
\operatorname{det}\left(\underline{\gamma_{\infty}}\left(b_{1}\right)-\underline{\gamma_{\infty}}\left(a_{1}\right), \ldots, \underline{\gamma_{\infty}}\left(b_{r}\right)-\underline{\gamma_{\infty}}\left(a_{r}\right)\right)=0
$$

for any $0 \leq a_{1}<b_{1} \leq \cdots \leq a_{r}<b_{r} \leq 1$. 
Let $S$ be the set of differentiability points $t \in(0,1)$ of $\gamma_{\infty}$ and let

$$
\mathfrak{h}_{1}:=\operatorname{span}\left\{\underline{\dot{\gamma}_{\infty}}(t) \mid t \in S\right\} \subseteq \mathfrak{g}_{1}
$$

be the linear subspace of $\mathfrak{g}_{1}$ spanned by the derivatives $\dot{\gamma}_{\infty}(t)$. We claim that $\operatorname{dim} \mathfrak{h}_{1}<$ $r$. If this were not the case, we could find $0<t_{1}<\cdots<t_{r}<1, t_{i} \in S$, such that $\underline{\dot{\gamma}_{\infty}}\left(t_{1}\right), \ldots, \dot{\gamma}_{\infty}\left(t_{r}\right)$ are linearly independent. Setting

$$
a_{i}:=t_{i}, b_{i}:=t_{i}+\delta, \quad i=1, \ldots, r
$$

and letting $\delta \downarrow 0$ in (2.10), we would deduce that $\operatorname{det}\left(\underline{\dot{\gamma}_{\infty}}\left(t_{1}\right), \ldots, \underline{\dot{\gamma}_{\infty}}\left(t_{r}\right)\right)=0$, which is a contradiction.

As a consequence, there exists a unit vector $v \in \mathfrak{g}_{1}$ orthogonal to $\mathfrak{h}_{1}$ and we obtain

$$
\operatorname{Exc}\left(\gamma_{\infty} ;[0,1]\right) \leq\left(\int_{0}^{1}\left\langle v, \underline{\dot{\gamma}}_{\infty}(t)\right\rangle^{2} d t\right)^{1 / 2}=0
$$

But from $\operatorname{Exc}\left(\gamma_{k_{p}} ;[0,1]\right) \geq \varepsilon$ and $\operatorname{Remark} 2.5$ we also have $\operatorname{Exc}\left(\gamma_{\infty} ;[0,1]\right) \geq \varepsilon$. This is a contradiction and the proof is accomplished.

Remark 2.8. Under the same assumptions and notation of Lemma 2.7, we also have

$$
\left|\underline{\gamma}\left(b_{i}\right)-\underline{\gamma}\left(a_{i}\right)\right| \geq c \mathscr{L}^{1}(I) \quad \text { for any } i=1, \ldots, r .
$$

Indeed, one has $\left|\underline{\gamma}\left(b_{i}\right)-\underline{\gamma}\left(a_{i}\right)\right| \leq \mathscr{L}^{1}(I)$ by arclength parametrization and (2.8) could not hold in case (2.11) were false for some index $i$.

\section{Cut And CORRECTION DEvices}

In this section we introduce the cut and the iterated correction of a horizontal curve. In Lemma 3.4 we compute the gain of length in terms of the excess. In the formula (3.16), we establish an algebraic identity for the displacement of the endpoint produced by an iterated correction. We keep on working in a Carnot group G.

The concatenation of two curves $\alpha:\left[a, a+a^{\prime}\right] \rightarrow G$ and $\beta:\left[b, b+b^{\prime}\right] \rightarrow G$ is the curve $\alpha * \beta:\left[a, a+\left(a^{\prime}+b^{\prime}\right)\right] \rightarrow G$ defined by the formula

$$
\alpha * \beta(t):= \begin{cases}\alpha(t) & \text { if } t \in\left[a, a+a^{\prime}\right] \\ \alpha\left(a+a^{\prime}\right) \beta(b)^{-1} \beta\left(t+b-\left(a+a^{\prime}\right)\right) & \text { if } t \in\left[a+a^{\prime}, a+a^{\prime}+b^{\prime}\right] .\end{cases}
$$

The concatenation $\alpha * \beta$ is continuous if $\alpha$ and $\beta$ are continuous and it is horizontal if $\alpha$ and $\beta$ are horizontal. The operation $*$ is associative.

Definition 3.1 (Cut curve). Let $\gamma:[a, b] \rightarrow G$ be a curve. For any subinterval $\left[s, s^{\prime}\right] \subseteq[a, b]$ with $\underline{\gamma}\left(s^{\prime}\right) \neq \underline{\gamma}(s)$ we define the cut curve $\operatorname{Cut}\left(\gamma ;\left[s, s^{\prime}\right]\right):\left[a, b^{\prime \prime}\right] \rightarrow G$, with $b^{\prime \prime}:=b-\left(s^{\prime}-s\right)+\left|\underline{\gamma}\left(s^{\prime}\right)-\underline{\gamma}(s)\right|$, by the formula

$$
\operatorname{Cut}\left(\gamma ;\left[s, s^{\prime}\right]\right):=\left.\left.\left.\gamma\right|_{[a, s]} * \exp (\cdot w)\right|_{\left[0,\left|\underline{\gamma}\left(s^{\prime}\right)-\underline{\gamma}(s)\right|\right]} * \gamma\right|_{\left[s^{\prime}, b\right]},
$$


where

$$
w:=\frac{\underline{\gamma}\left(s^{\prime}\right)-\underline{\gamma}(s)}{\left|\underline{\gamma}\left(s^{\prime}\right)-\underline{\gamma}(s)\right|} .
$$

When $\underline{\gamma}\left(s^{\prime}\right)=\underline{\gamma}(s)$, the cut curve is defined by

$$
\operatorname{Cut}\left(\gamma ;\left[s, s^{\prime}\right]\right)=\left.\left.\gamma\right|_{[a, s]} * \gamma\right|_{\left[s^{\prime}, b\right]} \cdot
$$

Remark 3.2. If $\gamma$ is parametrized by arclength and horizontal, then the cut curve $\operatorname{Cut}\left(\gamma ;\left[s, s^{\prime}\right]\right)$ is parametrized by arclength and horizontal, with length

$$
L\left(\operatorname{Cut}\left(\gamma ;\left[s, s^{\prime}\right]\right)\right)=L(\gamma)-\left(s^{\prime}-s\right)+\left|\underline{\gamma}\left(s^{\prime}\right)-\underline{\gamma}(s)\right| .
$$

Remark 3.3. The final point of the cut curve has the same projection on $\mathfrak{g}_{1}$ as the final point of $\gamma$, i.e., $\pi\left(\operatorname{Cut}\left(\gamma ;\left[s, s^{\prime}\right]\right)\left(b^{\prime \prime}\right)\right)=\pi(\gamma(b))$. Indeed, by Lemma 3.6 below we have

$$
\begin{aligned}
\pi\left(\operatorname{Cut}\left(\gamma ;\left[s, s^{\prime}\right]\right)\left(b^{\prime \prime}\right)\right) & =\pi\left(\gamma(s) \exp \left(\left|\underline{\gamma}\left(s^{\prime}\right)-\underline{\gamma}(s)\right| w\right) \gamma\left(s^{\prime}\right)^{-1} \gamma(b)\right) \\
& =\underline{\gamma}(s)+\left|\underline{\gamma}\left(s^{\prime}\right)-\underline{\gamma}(s)\right| w+\left(\underline{\gamma}(b)-\underline{\gamma}\left(s^{\prime}\right)\right) \\
& =\underline{\gamma}(b) .
\end{aligned}
$$

Lemma 3.4. Let $\gamma: I \rightarrow G$ be a horizontal curve parametrized by arclength on a compact interval $I$ and let $J \subseteq I$ be a subinterval with $\mathscr{L}^{1}(J)>0$. Then we have

$$
L(\gamma)-L(\operatorname{Cut}(\gamma ; J)) \geq \frac{\mathscr{L}^{1}(J)}{2} \operatorname{Exc}(\gamma ; J)^{2} .
$$

Proof. Let $J=\left[s, s^{\prime}\right]$ for some $s<s^{\prime}$. As in Definition [3.1, let $w \in \mathfrak{g}_{1}$ be a unit vector such that $\left\langle w, \underline{\gamma}\left(s^{\prime}\right)-\underline{\gamma}(s)\right\rangle=\left|\underline{\gamma}\left(s^{\prime}\right)-\underline{\gamma}(s)\right|$, i.e.,

$$
\left\langle w, f_{s}^{s^{\prime}} \dot{\gamma}(t) d t\right\rangle=\frac{\left|\underline{\gamma}\left(s^{\prime}\right)-\underline{\gamma}(s)\right|}{s^{\prime}-s} .
$$

Since $|\underline{\dot{\gamma}}|=1$ a.e., we have $|\underline{\dot{\gamma}}-w|^{2}=2(1-\langle w, \underline{\dot{\gamma}}\rangle)$, and since $r \geq 2$ there exists a unit vector $v \in \mathfrak{g}_{1}$ with $\langle v, w\rangle=0$. Thus, for all $t$ such that $\underline{\dot{\gamma}}(t)$ is defined we have

$$
|\langle v, \underline{\dot{\gamma}}(t)\rangle|=|\langle v, \dot{\gamma}(t)-w\rangle| \leq|\underline{\dot{\gamma}}(t)-w| .
$$

We deduce that

$$
\begin{aligned}
\operatorname{Exc}\left(\gamma ;\left[s, s^{\prime}\right]\right)^{2} & \leq f_{s}^{s^{\prime}}\langle v, \underline{\dot{\gamma}}(t)\rangle^{2} d t \leq f_{s}^{s^{\prime}}|\dot{\gamma}(t)-w|^{2} d t \\
& =2\left(1-\left\langle w, f_{s}^{s^{\prime}} \dot{\underline{\gamma}}(t) d t\right\rangle\right)=2\left(1-\frac{\left|\underline{\gamma}\left(s^{\prime}\right)-\underline{\gamma}(s)\right|}{s^{\prime}-s}\right) .
\end{aligned}
$$

Multiplying by $\mathscr{L}^{1}(J)=s^{\prime}-s$ and using (3.12), we obtain the claim:

$$
\mathscr{L}^{1}(J) \operatorname{Exc}(\gamma ; J)^{2} \leq 2\left(\left(s^{\prime}-s\right)-\left|\underline{\gamma}\left(s^{\prime}\right)-\underline{\gamma}(s)\right|\right)=2(L(\gamma)-L(\operatorname{Cut}(\gamma ; J))) .
$$


Given $Y \in \mathfrak{g}$, we hereafter denote by $\delta_{Y}:\left[0, \ell_{Y}\right] \rightarrow G$ any geodesic from 0 to $\exp (Y)$ parametrized by arclength (the choice of $\delta_{Y}$ is not important); in particular, $\ell_{Y}=d(0, \exp (Y))$. We denote by $\delta_{Y}\left(\ell_{Y}-\cdot\right)$ the curve $\delta_{Y}$ traveled backwards from $\exp (Y)$ to 0 .

Definition 3.5 (Corrected curve and displacement). Let $\gamma:[a, b] \rightarrow G$ be a horizontal curve parametrized by arclength. For any subinterval $\left[s, s^{\prime}\right] \subseteq[a, b]$ and $Y \in \mathfrak{g}$, we define the corrected curve $\operatorname{Cor}\left(\gamma ;\left[s, s^{\prime}\right], Y\right):\left[a, b^{\prime \prime \prime}\right] \rightarrow G$, with $b^{\prime \prime \prime}:=b+2 \ell_{Y}$, by

$$
\operatorname{Cor}\left(\gamma ;\left[s, s^{\prime}\right], Y\right):=\left.\left.\left.\gamma\right|_{[a, s]} * \delta_{Y} * \gamma\right|_{\left[s, s^{\prime}\right]} * \delta_{Y}\left(\ell_{Y}-\cdot\right) * \gamma\right|_{\left[s^{\prime}, b\right]} \cdot
$$

We refer to the process of transforming $\gamma$ into $\operatorname{Cor}\left(\gamma ;\left[s, s^{\prime}\right], Y\right)$ as to the application of the correction device associated with $\left[s, s^{\prime}\right]$ and $Y$. The displacement of the final point produced by the correction device associated with $\left[s, s^{\prime}\right]$ and $Y$ is

$$
\operatorname{Dis}\left(\gamma ;\left[s, s^{\prime}\right], Y\right):=\gamma(b)^{-1} \operatorname{Cor}\left(\gamma ;\left[s, s^{\prime}\right], Y\right)\left(b^{\prime \prime \prime}\right)
$$

We will later express the displacement in terms of suitable conjugations $C_{g}(h):=$ $g h g^{-1}$ and commutators $[g, h]:=g h g^{-1} h^{-1}$ in $G$.

For any $1 \leq j \leq s$, we denote by $\bar{\pi}_{j}: \mathfrak{g} \rightarrow \mathfrak{g}_{j}$ the canonical projection with respect to the direct sum. The mappings $\pi_{j}: G \rightarrow \mathfrak{g}$ are defined as $\pi_{j}:=\bar{\pi}_{j} \circ \exp ^{-1}$. Clearly, one has $\bar{\pi}_{1}=\bar{\pi}$ and $\pi_{1}=\pi$. We let $\mathfrak{w}_{j}:=\mathfrak{g}_{j} \oplus \cdots \oplus \mathfrak{g}_{s}$ and $G_{j}:=\exp \left(\mathfrak{w}_{j}\right)$. We also agree that $G_{s+1}:=\{0\}$, the identity element of $G$, and $\mathfrak{w}_{s+1}:=\{0\}$.

Lemma 3.6. The map $\pi: G \rightarrow\left(\mathfrak{g}_{1},+\right)$ is a group homomorphism and

$$
\pi_{*} X_{i}=X_{i} \quad \text { for } i=1, \ldots, r .
$$

For any $1 \leq j \leq s, G_{j}$ is a subgroup of $G$ and $\pi_{j}: G_{j} \rightarrow\left(\mathfrak{g}_{j},+\right)$ is a group homomorphism.

Proof. Given points $g=\exp \left(x_{1} X_{1}+\cdots+x_{n} X_{n}\right)$ and $g^{\prime}=\exp \left(x_{1}^{\prime} X_{1}+\cdots+x_{n}^{\prime} X_{n}\right)$ in $G$, by (2.4) we have $\exp ^{-1}\left(g g^{\prime}\right)=\left(x_{1}+x_{1}^{\prime}\right) X_{1}+\cdots+\left(x_{r}+x_{r}^{\prime}\right) X_{r}+R$ with $R \in \mathfrak{w}_{2}$ and hence

$$
\pi\left(g g^{\prime}\right)=\bar{\pi}\left(\exp ^{-1}\left(g g^{\prime}\right)\right)=\left(x_{1}+x_{1}^{\prime}\right) X_{1}+\cdots+\left(x_{r}+x_{r}^{\prime}\right) X_{r}=\pi(g)+\pi\left(g^{\prime}\right) .
$$

The identity (3.13) follows from this and the left-invariance of $X_{i}$. The fact that $G_{j}$ is a subgroup follows from the Baker-Campbell-Hausdorff formula, and the assertion that $\pi_{j}: G_{j} \rightarrow \mathfrak{g}_{j}$ is a homomorphism can be obtained as above.

The following lemmas describe how the homomorphisms $\pi_{j}$ interact with conjugations, commutators and Lie brackets. We denote by $\operatorname{Ad}(g)$ the differential of the conjugation $C_{g}$ at the identity $0 \in G$. This is an automorphism of $T_{0} G=\mathfrak{g}$. For $X, Y \in \mathfrak{g}$ and $g \in G$, we have the formulas $\operatorname{Ad}(\exp (X))=\mathrm{e}^{\operatorname{ad}(X)}$ and $C_{g}(\exp (Y))=$ $\exp (\operatorname{Ad}(g) Y)$, see e.g. [10, Proposition 1.91]. 
Lemma 3.7. For any $g \in G$ and $h \in G_{j}$ we have $g h g^{-1} \in G_{j}$ (i.e., $G_{j}$ is normal in $G)$ and $\pi_{j}\left(g h g^{-1}\right)=\pi_{j}(h)$.

Proof. With $g=\exp (X)$ and $h=\exp (Y)$, we have

$$
\exp ^{-1}\left(g h g^{-1}\right)=\operatorname{Ad}(g) Y=\mathrm{e}^{\operatorname{ad} X} Y=\sum_{k=0}^{\infty} \frac{(\operatorname{ad} X)^{k}}{k !} Y=Y+R,
$$

with $R \in \mathfrak{w}_{j+1}$, because in the previous sum all the terms with $k \geq 1$ belong to $\mathfrak{w}_{j+1}$. Hence, we have $g h g^{-1} \in G_{j}$ and

$$
\pi_{j}\left(g h g^{-1}\right)=\bar{\pi}_{j} \circ \exp ^{-1}\left(g h g^{-1}\right)=\bar{\pi}_{j}(Y+R)=\bar{\pi}_{j}(Y)=\pi_{j}(h) .
$$

Lemma 3.8. For any $g \in G$ and $h \in G_{j}$ with $1 \leq j<s$ we have

$$
[g, h] \in G_{j+1} \quad \text { and } \quad \pi_{j+1}([g, h])=\left[\pi(g), \pi_{j}(h)\right] .
$$

A similar statement holds if $g \in G_{j}$ and $h \in G$.

Proof. We prove only the first part of the statement, the second one following from the first one and the identity $[g, h]=[h, g]^{-1}$. Combining Lemma 3.7 with Lemma 3.6, we obtain $[g, h]=\left(g h g^{-1}\right) h^{-1} \in G_{j}$ and

$$
\pi_{j}([g, h])=\pi_{j}\left(g h g^{-1}\right)+\pi_{j}\left(h^{-1}\right)=\pi_{j}(h)-\pi_{j}(h)=0,
$$

so that $[g, h] \in G_{j+1}$. Now, writing $g=\exp (X), h=\exp (Y)$ and using the formula $\exp ^{-1}\left(g h g^{-1}\right)=\mathrm{e}^{\text {ad } X} Y$ as in the previous proof, we obtain

$$
\exp ^{-1}\left(g h g^{-1}\right)=\sum_{k=0}^{\infty} \frac{(\operatorname{ad} X)^{k}}{k !} Y=Y+[X, Y]+R^{\prime},
$$

where the remainder $R^{\prime}$ is the sum of all terms with $k \geq 2$ and thus belongs to $\mathfrak{w}_{j+2}$. As $h^{-1}=\exp (-Y)$, the Baker-Campbell-Hausdorff formula gives

$$
\exp ^{-1}([g, h])=P\left(Y+[X, Y]+R^{\prime},-Y\right)=[X, Y]+R^{\prime}+R^{\prime \prime},
$$

where $R^{\prime \prime}$ is given by the commutators of length at least 2 appearing in (2.4). Now, thinking each such commutator as a $\left(k_{1}+\ell_{1}+\cdots+k_{p}+\ell_{p}\right)$-multilinear function (and expanding each instance of $Y+[X, Y]+R^{\prime}$ accordingly), we obtain that $R^{\prime \prime}$ is a linear combination of elements of the form

$$
\left(\operatorname{ad} Z_{1}\right) \cdots\left(\operatorname{ad} Z_{k}\right) Z_{k+1}
$$

where $k \geq 1$ and $Z_{i} \in\left\{Y,[X, Y], R^{\prime}\right\}$. Those elements where only $Y$ appears vanish, while the other terms belong to $\mathfrak{w}_{j+2}$, since $[X, Y], R^{\prime} \in \mathfrak{w}_{j+1}$ and $k \geq 1$. We deduce that $R^{\prime \prime} \in \mathfrak{w}_{j+2}$. Finally,

$$
\pi_{j+1}([g, h])=\bar{\pi}_{j+1}\left([X, Y]+R^{\prime}+R^{\prime \prime}\right)=\bar{\pi}_{j+1}([X, Y])=\left[\bar{\pi}(X), \bar{\pi}_{j}(Y)\right],
$$

since $X=\bar{\pi}(X)+R_{X}$ and $Y=\bar{\pi}_{j}(Y)+R_{Y}$, with $R_{X} \in \mathfrak{w}_{2}$ and $R_{Y} \in \mathfrak{w}_{j+1}$. 
Hereafter, we adopt the short notation $\left.\gamma\right|_{a} ^{b}:=\gamma(a)^{-1} \gamma(b)$.

Lemma 3.9. Under the assumptions and notation of Definition 3.5, the displacement is given by the formula

$$
\operatorname{Dis}\left(\gamma ;\left[s, s^{\prime}\right], Y\right)=C_{\left.\gamma\right|_{b} ^{s}}\left(\left[\exp (Y),\left.\gamma\right|_{s} ^{s^{\prime}}\right]\right) \text {. }
$$

In particular, if $Y \in \mathfrak{g}_{j}$ and $1 \leq j<s$, then $\operatorname{Dis}\left(\gamma ;\left[s, s^{\prime}\right], Y\right) \in G_{j+1}$ and

$$
\pi_{j+1}\left(\operatorname{Dis}\left(\gamma ;\left[s, s^{\prime}\right], Y\right)\right)=\left[Y, \underline{\gamma}\left(s^{\prime}\right)-\underline{\gamma}(s)\right] .
$$

Proof. We have

$$
\begin{aligned}
\operatorname{Cor}\left(\gamma ;\left[s, s^{\prime}\right], Y\right)\left(b^{\prime \prime \prime}\right) & =\left.\left.\gamma(s) \exp (Y) \gamma\right|_{s} ^{s^{\prime}} \exp (-Y) \gamma\right|_{s^{\prime}} ^{b} \\
& =\left.\left.\gamma(s)\left[\exp (Y),\left.\gamma\right|_{s} ^{s^{\prime}}\right] \gamma\right|_{s} ^{s^{\prime}} \gamma\right|_{s^{\prime}} ^{b} \\
& =\left.\gamma(s)\left[\exp (Y),\left.\gamma\right|_{s} ^{s^{\prime}}\right] \gamma\right|_{s} ^{b},
\end{aligned}
$$

hence

$$
\left.\operatorname{Dis}\left(\gamma ;\left[s, s^{\prime}\right], Y\right)\right)=\left.\gamma\right|_{b} ^{s}\left[\exp (Y),\left.\gamma\right|_{s} ^{s^{\prime}}\right]\left(\left.\gamma\right|_{b} ^{s}\right)^{-1}=C_{\left.\gamma\right|_{b} ^{s}}\left(\left[\exp (Y),\left.\gamma\right|_{s} ^{s^{\prime}}\right]\right) .
$$

By Lemma 3.6, we have $\pi\left(\left.\gamma\right|_{s} ^{s^{\prime}}\right)=\underline{\gamma}\left(s^{\prime}\right)-\underline{\gamma}(s)$; moreover, $\pi_{j}(\exp (Y))=Y$. Hence, using Lemma 3.8, we obtain

$$
\left[\exp (Y),\left.\gamma\right|_{s} ^{s^{\prime}}\right] \in G_{j+1} \quad \text { and } \quad \pi_{j+1}\left(\left[\exp (Y),\left.\gamma\right|_{s} ^{s^{\prime}}\right]\right)=\left[Y, \underline{\gamma}\left(s^{\prime}\right)-\underline{\gamma}(s)\right]
$$

The lemma now follows from equation (3.14) and Lemma 3.7.

Definition 3.10 (Iterated correction). Let $\gamma: I \rightarrow G$ be a horizontal curve parametrized by arclength on the interval $I$ and let $I_{1}:=\left[s_{1}, t_{1}\right], \ldots, I_{k}:=\left[s_{k}, t_{k}\right] \subseteq I$ be subintervals with $t_{i} \leq s_{i+1}$. For any $Y_{1}, \ldots, Y_{k} \in \mathfrak{g}$ we define by induction on $k \geq 2$ the iterated correction

$$
\operatorname{Cor}\left(\gamma ; I_{1}, Y_{1} ; \ldots ; I_{k}, Y_{k}\right):=\operatorname{Cor}\left(\operatorname{Cor}\left(\gamma ; I_{1}, Y_{1} ; \ldots ; I_{k-1}, Y_{k-1}\right) ; I_{k}+2 \sum_{i<k} \ell_{Y_{i}}, Y_{k}\right) .
$$

The iterated correction is a curve defined on the interval $[a, \widehat{b}]$, with $\widehat{b}:=b+$ $2 \sum_{i=1}^{k} \ell_{Y_{i}}$. The displacement of the final point produced by this iterated correction is

$$
\operatorname{Dis}\left(\gamma ; I_{1}, Y_{1} ; \ldots ; I_{k}, Y_{k}\right):=\gamma(b)^{-1} \operatorname{Cor}\left(\gamma ; I_{1}, Y_{1} ; \ldots ; I_{k}, Y_{k}\right)(\widehat{b})
$$

Corollary 3.11. For any $I_{i}=\left[s_{i}, t_{i}\right] \subseteq I$ and $Y_{i} \in \mathfrak{g}_{j}$, with $i=1, \ldots, k$ and $j<s$, we have

$$
\operatorname{Dis}\left(\gamma ; I_{1}, Y_{1} ; \ldots ; I_{k}, Y_{k}\right) \in G_{j+1}
$$

and

$$
\pi_{j+1}\left(\operatorname{Dis}\left(\gamma ; I_{1}, Y_{1} ; \ldots ; I_{k}, Y_{k}\right)\right)=\sum_{i=1}^{k}\left[Y_{i}, \underline{\gamma}\left(t_{i}\right)-\underline{\gamma}\left(s_{i}\right)\right]
$$


Proof. We prove (3.16) by induction on $k$. The case $k=1$ is in Lemma 3.9, Assume the formula holds for $k-1$. Letting $\widehat{\gamma}:=\operatorname{Cor}\left(\gamma ; I_{1}, Y_{1} ; \ldots ; I_{k-1}, Y_{k-1}\right)$, which is defined on the interval $[a, \widehat{b}]$ (where $\widehat{b}:=b+2 \sum_{i<k} \ell_{Y_{i}}$ ), we have

$$
\begin{aligned}
\operatorname{Dis}\left(\gamma ; I_{1}, Y_{1} ; \ldots ; I_{k}, Y_{k}\right) & =\gamma(b)^{-1} \operatorname{Cor}\left(\widehat{\gamma} ; I_{k}+(\widehat{b}-b), Y_{k}\right) \\
& =\gamma(b)^{-1} \widehat{\gamma}(\widehat{b}) \operatorname{Dis}\left(\widehat{\gamma} ; I_{k}+(\widehat{b}-b), Y_{k}\right) \\
& =\operatorname{Dis}\left(\gamma ; I_{1}, Y_{1} ; \ldots ; I_{k-1}, Y_{k-1}\right) \operatorname{Dis}\left(\widehat{\gamma} ; I_{k}+(\widehat{b}-b), Y_{k}\right)
\end{aligned}
$$

Then, by Lemma 3.6, by the inductive assumption and by Lemma 3.9 applied to $\widehat{\gamma}$ we have

$$
\begin{aligned}
& \pi_{j+1}\left(\operatorname{Dis}\left(\gamma ; I_{1}, Y_{1} ; \ldots ; I_{k}, Y_{k}\right)\right) \\
= & \sum_{i=1}^{k-1}\left[Y_{i}, \underline{\gamma}\left(t_{i}\right)-\underline{\gamma}\left(s_{i}\right)\right]+\left[Y_{k}, \widehat{\gamma}\left(t_{k}+(\widehat{b}-b)\right)-\widehat{\underline{\gamma}}\left(s_{k}+(\widehat{b}-b)\right)\right] \\
= & \sum_{i=1}^{k}\left[Y_{i}, \underline{\gamma}\left(t_{i}\right)-\underline{\gamma}\left(s_{i}\right)\right]
\end{aligned}
$$

because $\underline{\hat{\gamma}}\left(t_{k}+(\widehat{b}-b)\right)-\underline{\widehat{\gamma}}\left(s_{k}+(\widehat{b}-b)\right)=\underline{\gamma}\left(t_{k}\right)-\underline{\gamma}\left(s_{k}\right)$.

When dealing with curves $\gamma$ defined on symmetric intervals, it is convenient to use modified versions of Cut and Cor, which we will denote by $\operatorname{Cut}^{\prime}\left(\gamma ;\left[s, s^{\prime}\right]\right)$ and $\operatorname{Cor}^{\prime}\left(\gamma ;\left[s, s^{\prime}\right], Y\right)$. They are obtained from $\operatorname{Cut}\left(\gamma ;\left[s, s^{\prime}\right]\right)$ and $\operatorname{Cor}\left(\gamma ;\left[s, s^{\prime}\right], Y\right)$ by composition with the time translation such that the new domain is a symmetric interval. The iterated correction is then defined in the following way:

$$
\operatorname{Cor}^{\prime}\left(\gamma ; I_{1}, Y_{1}, \ldots ; I_{k}, Y_{k}\right):=\operatorname{Cor}^{\prime}\left(\operatorname{Cor}^{\prime}\left(\gamma ; I_{1}, Y_{1} ; \ldots ; I_{k-1}, Y_{k-1}\right) ; I_{k}+\sum_{i<k} \ell_{Y_{i}}, Y_{k}\right) .
$$

The related displacement satisfies the properties (3.15) and (3.16) of Corollary 3.11 with Cor' $^{\prime}$ replacing Cor.

\section{Proof of the main Results}

Let $G$ be a Carnot group with rank $r \geq 2$ and step $s$, and let $\mathscr{X}=\left\{X_{1}, \ldots, X_{r}\right\}$ be an orthonormal basis for $\mathfrak{g}_{1}$ (recall that $\mathfrak{g}$ is endowed with a scalar product such that $\mathfrak{g}_{i} \perp \mathfrak{g}_{j}$ ). We first prove the one-sided version of Theorem 1.2, we will illustrate later how to adapt the proof in order to obtain Theorem 1.2 .

Theorem 4.1. Let $\gamma:[0, T] \rightarrow G, T>0$, be a length-minimizing curve parametrized by arclength. Then there exists an infinitesimal sequence $\eta_{i} \downarrow 0$ such that

$$
\lim _{i \rightarrow \infty} \operatorname{Exc}\left(\gamma ;\left[0, \eta_{i}\right]\right)=0
$$

Proof. Step 1. We can assume that $\gamma(0)=0$. Suppose by contradiction that there exists $\varepsilon>0$ such that $\operatorname{Exc}(\gamma ;[0, t]) \geq \varepsilon$ for any sufficiently small $t>0$. For $k=$ 
$1, \ldots, s$, we inductively define horizontal curves $\gamma^{(k)}:\left[0, T_{k}\right] \rightarrow G$ parametrized by arclength such that:

(i) $\gamma^{(k)}(0)=\gamma(0)=0$;

(ii) $\gamma(T)^{-1} \gamma^{(k)}\left(T_{k}\right) \in G_{k+1}$, where $G_{s+1}=\{0\}$;

(iii) $L\left(\gamma^{(k)}\right)<L(\gamma)$, i.e., $T_{k}<T$.

In particular, $\gamma^{(s)}$ is a horizontal curve with the same endpoints as $\gamma$, but with smaller length: this contradicts the minimality of $\gamma$.

We define $\gamma^{(1)}:=\operatorname{Cut}(\gamma ;[0, \eta])$, where the parameter $\eta>0$ will be chosen later; in fact, any sufficiently small $\eta$ will work. In this proof, the notation $O(\cdot)$ and $o(\cdot)$ is used for asymptotic estimates which hold as $\eta \rightarrow 0$. By Remark 3.3 and Lemma 3.4, $\gamma^{(1)}$ satisfies (i), (ii) and (iii) with $k=1$.

Step 2. Let us fix parameters $\beta>0$ and $\varrho_{1}:=1>\varrho_{2}>\cdots>\varrho_{s}>0$ such that for all $k=1, \ldots, s-1$

$$
\frac{(k+1) \varrho_{k}-\varrho_{k+1}}{k}>1+\beta .
$$

This is possible if $\beta$ is small enough: indeed, the inequality (4.18) is equivalent to

$$
\varrho_{k}>\frac{\varrho_{k+1}+k}{k+1}+\frac{k}{k+1} \beta,
$$

and we can choose any $\varrho_{s} \in(0,1)$ and then $\varrho_{s-1}<1$ so as to verify the (strict) inequality when $\beta=0$ and $k=s-1$, then $\varrho_{s-2}$ similarly and so on, up to $\varrho_{1}=1$. By continuity, the inequalities will still hold for a small enough $\beta>0$.

For any $k=1, \ldots, s$, we set $I_{k}:=\left[0, \eta^{\varrho_{k}}\right]$; the curve $\gamma^{(k+1)}$ is defined from $\gamma^{(k)}$ by applying several correction devices within $I_{k+1}$, see (4.21). As soon as $\eta \leq 1$, the inclusions $[0, \eta]=I_{1} \subseteq I_{2} \subseteq \cdots \subseteq I_{s}$ hold.

By Lemma 3.4, since $\operatorname{Exc}(\gamma ;[0, \eta]) \geq \varepsilon$, the gain of length obtained by performing the cut is

$$
L(\gamma)-L\left(\gamma^{(1)}\right) \geq \frac{\eta \varepsilon^{2}}{2} \geq \eta^{1+\beta}
$$

provided $\eta$ is small enough.

The curves $\gamma^{(k)}:\left[0, T_{k}\right] \rightarrow G$ will be constructed inductively so as to satisfy (i), (ii) and (iii), as well as the following additional technical properties, which hold for $\gamma^{(1)}$ :

(iv) $T_{k} \geq T_{k-1}$ if $k \geq 2$;

(v) $L\left(\gamma^{(k)}\right) \leq L(\gamma)-(1+o(1)) \eta^{1+\beta}$;

(vi) $\underline{\gamma^{(k)}}(t)=\underline{\gamma}\left(t+\left(T-T_{k}\right)\right)$ for any $t \in\left[2 \eta^{\varrho_{k}}, T_{k}\right]$, i.e., on $\left[2 \eta^{\varrho_{k}}, T_{k}\right]$ the curve $\gamma^{(k)}$ has the same projection on $\mathfrak{g}_{1}$ as the corresponding final piece of $\gamma$;

(vii) $\left\|\underline{\gamma^{(k)}}-\left.\underline{\gamma}\right|_{\left[0, T_{k}\right]}\right\|_{L^{\infty}}=O(\eta)$.

Notice that (v) implies (iii) for small enough $\eta$. 
Step 3. Assume that $\gamma^{(k)}$ has been constructed, for some $1 \leq k \leq s-1$, in such a way that (i)-(vii) hold. By (ii), there exists $E_{k} \in \mathfrak{g}_{k+1} \oplus \cdots \oplus \mathfrak{g}_{s}$ such that

$$
\gamma(T)^{-1} \gamma^{(k)}\left(T_{k}\right)=\exp \left(E_{k}\right)
$$

Let us estimate $\bar{\pi}_{k+1}\left(E_{k}\right)$. First, by (vi) and the uniqueness of horizontal lifts, we have

$$
\left.\gamma^{(k)}\right|_{2 \eta^{\varrho_{k}}} ^{T_{k}}=\left.\gamma\right|_{\tau_{k}} ^{T}, \quad \text { where } \tau_{k}:=2 \eta^{\varrho_{k}}+\left(T-T_{k}\right) .
$$

Hence, defining $g_{k}:=\gamma\left(\tau_{k}\right)^{-1} \gamma^{(k)}\left(2 \eta^{\varrho_{k}}\right)$, we have

$$
\begin{aligned}
\gamma^{(k)}\left(T_{k}\right) & =\left.\gamma^{(k)}\left(2 \eta^{\varrho_{k}}\right) \gamma^{(k)}\right|_{2 \eta^{\varrho_{k}}} ^{T_{k}}=\left.\gamma\left(\tau_{k}\right) g_{k} \gamma\right|_{\tau_{k}} ^{T} \\
& =\left.\gamma\left(\tau_{k}\right) \gamma\right|_{\tau_{k}} ^{T} C_{\left.\gamma\right|_{T} ^{\tau_{k}}}\left(g_{k}\right)=\gamma(T) C_{\left.\gamma\right|_{T} ^{\tau_{k}}\left(g_{k}\right),}
\end{aligned}
$$

i.e., $g_{k}=C_{\gamma_{\tau_{k}}^{T}}\left(\gamma(T)^{-1} \gamma^{(k)}\left(T_{k}\right)\right)$. From (ii) and Lemma 3.7 we obtain $g_{k} \in G_{k+1}$ and

$$
\bar{\pi}_{k+1}\left(E_{k}\right)=\pi_{k+1}\left(\gamma(T)^{-1} \gamma^{(k)}\left(T_{k}\right)\right)=\pi_{k+1}\left(g_{k}\right)=O\left(\eta^{(k+1) \varrho_{k}}\right) .
$$

The last estimate follows from (2.6) applied to the curve

$$
\left.\left.\gamma\left(\tau_{k}\right)^{-1} \gamma\right|_{\left[0, \tau_{k}\right]}\left(\tau_{k}-\cdot\right) * \gamma^{(k)}\right|_{\left[0,2 \eta^{\varrho} k\right]},
$$

which connects 0 to $\gamma\left(\tau_{k}\right)^{-1} \gamma^{(k)}\left(2 \eta^{\varrho_{k}}\right)$. Its length is $\tau_{k}+2 \eta^{\varrho_{k}}$ and is controlled by $5 \eta^{\varrho_{k}}$ because, by (iv),

$$
T-T_{k} \leq T-T_{1}=L(\gamma)-L\left(\gamma^{(1)}\right) \leq \eta \leq \eta^{\varrho_{k}}
$$

Step 4. We now define $\gamma^{(k+1)}$. As $\mathfrak{g}_{k+1}=\left[\mathfrak{g}_{k}, \mathfrak{g}_{1}\right]$, using estimate (4.19) for $\bar{\pi}_{k+1}\left(E_{k}\right)$, there exist $Y_{1}, \ldots, Y_{r} \in \mathfrak{g}_{k}$ such that

$$
\bar{\pi}_{k+1}\left(E_{k}\right)=\sum_{i=1}^{r}\left[Y_{i}, X_{i}\right] \text { and }\left|Y_{1}\right|, \ldots,\left|Y_{r}\right|=O\left(\eta^{(k+1) \varrho_{k}}\right) .
$$

Furthermore, we have $\operatorname{Exc}\left(\gamma ; I_{k+1}\right) \geq \varepsilon$ whenever $\eta$ is small enough. We can then apply Lemma 2.7 to $I_{k+1}$ and find $\left[a_{1}, b_{1}\right], \ldots,\left[a_{r}, b_{r}\right] \subseteq I_{k+1}$ (with $b_{i} \leq a_{i+1}$ ) such that

$$
\left|\operatorname{det}\left(\underline{\gamma}\left(b_{1}\right)-\underline{\gamma}\left(a_{1}\right), \ldots, \underline{\gamma}\left(b_{r}\right)-\underline{\gamma}\left(a_{r}\right)\right)\right| \geq c \eta^{r \varrho_{k+1}} .
$$

Using (vii) we obtain, for small $\eta$,

$$
\begin{aligned}
\left|\operatorname{det}\left(\underline{\gamma^{(k)}}\left(b_{1}\right)-\underline{\gamma^{(k)}}\left(a_{1}\right), \ldots, \underline{\gamma^{(k)}}\left(b_{r}\right)-\underline{\gamma^{(k)}}\left(a_{r}\right)\right)\right| & \geq c \eta^{r \varrho_{k+1}}-O\left(\eta^{1+(r-1) \varrho_{k+1}}\right) \\
& \geq \frac{c}{2} \eta^{r \varrho_{k+1}} .
\end{aligned}
$$

This implies that for $i=1, \ldots, r$ we have

$$
\left.X_{i}=\sum_{j=1}^{r} c_{i j} \underline{\left(\gamma^{(k)}\right.}\left(b_{j}\right)-\underline{\gamma^{(k)}}\left(a_{j}\right)\right) \text {, }
$$


where $\left|c_{i j}\right|=O\left(\eta^{-\varrho_{k+1}}\right)$. This estimate depends on $c$ and thus on $\varepsilon$. So, defining $Z_{j}=\sum_{i=1}^{r} c_{i j} Y_{i}$, from (4.20) we obtain

$$
\bar{\pi}_{k+1}\left(E_{k}\right)=\sum_{j=1}^{r}\left[Z_{j}, \underline{\gamma^{(k)}}\left(b_{j}\right)-\underline{\gamma^{(k)}}\left(a_{j}\right)\right]
$$

with $\left|Z_{j}\right|=O\left(\eta^{(k+1) \varrho_{k}-\varrho_{k+1}}\right)$. Finally, we let

$$
\gamma^{(k+1)}:=\operatorname{Cor}\left(\gamma^{(k)} ;\left[a_{1}, b_{1}\right],-Z_{1} ; \ldots ;\left[a_{r}, b_{r}\right],-Z_{r}\right) .
$$

Since $d(0, \exp (Z))=O\left(|Z|^{1 / k}\right)$ for $Z \in \mathfrak{g}_{k}$, the extra length $T_{k+1}-T_{k}$ needed for the application of these $r$ correction devices is

$$
T_{k+1}-T_{k}=\sum_{j=1}^{r} O\left(\left|Z_{j}\right|^{1 / k}\right)=O\left(\eta^{\frac{(k+1) \varrho_{k}-\varrho_{k+1}}{k}}\right)=o\left(\eta^{1+\beta}\right),
$$

thanks to the inequalities (4.18) on the parameters $\varrho_{k}$. Thus, we obtain

$$
L\left(\gamma^{(k+1)}\right) \leq L\left(\gamma^{(k)}\right)+o\left(\eta^{1+\beta}\right) .
$$

Step 5. We check that $\gamma^{(k+1)}$ satisfies properties (i)-(vii). We have just verified (iii) and (v), while (i) and (iv) are trivial. The property (vii) follows from the fact that $\gamma^{(k+1)}$ (as well as $\underline{\gamma^{(k+1)}}$ ) is obtained from $\gamma^{(k)}$ (from $\underline{\gamma^{(k)}}$ ) by the application of correction devices of total length $o\left(\eta^{1+\beta}\right)=O(\eta)$.

In order to check (vi), we remark that

$$
\underline{\gamma^{(k+1)}}=\left.\left.\underline{\gamma^{(k+1)}}\right|_{\left[0, \eta^{\varrho_{k+1}}+\left(T_{k+1}-T_{k}\right)\right]} * \underline{\gamma^{(k)}}\right|_{\left[\eta^{\varrho_{k+1}, T_{k}}\right]}
$$

and that the final point of the first curve in this concatenation coincides with the starting point of the second one. Since $T_{k+1}-T_{k}=O\left(\eta^{\frac{(k+1) \varrho_{k}-\varrho_{k+1}}{k}}\right)=o\left(\eta^{\varrho_{k+1}}\right)$, if $\eta$ is small enough we obtain

$$
\begin{aligned}
{\underline{\gamma^{(k+1)}}}_{\left[2 \eta^{\left.\varrho_{k+1}, T_{k+1}\right]}\right.} & =\left.\underline{\gamma^{(k)}}\right|_{\left[2 \eta^{\varrho_{k+1}}-\left(T_{k+1}-T_{k}\right), T_{k}\right]}\left(\cdot-\left(T_{k+1}-T_{k}\right)\right) \\
& =\left.\underline{\gamma}\right|_{\left[2 \eta^{\varrho_{k+1}}+\left(T-T_{k+1}\right), T\right]}\left(\cdot+\left(T-T_{k+1}\right)\right),
\end{aligned}
$$

the last equality holding by hypothesis (vi) for $k$, because $2 \eta^{\varrho_{k+1}}-\left(T_{k+1}-T_{k}\right) \geq 2 \eta^{\varrho_{k}}$ when $\eta$ is small. Thus, $\gamma^{(k+1)}$ satisfies (vi).

Finally, let us check (ii). By Lemma 3.6 and Corollary 3.11, we have

$$
\gamma(T)^{-1} \gamma^{(k+1)}\left(T_{k+1}\right)=\left(\gamma(T)^{-1} \gamma^{(k)}\left(T_{k}\right)\right)\left(\gamma^{(k)}\left(T_{k}\right)^{-1} \gamma^{(k+1)}\left(T_{k+1}\right)\right) \in G_{k+1}
$$

and

$$
\begin{aligned}
\pi_{k+1}\left(\gamma(T)^{-1} \gamma^{(k+1)}\left(T_{k+1}\right)\right) & =\pi_{k+1}\left(\exp \left(E_{k}\right)\right)+\pi_{k+1}\left(\gamma^{(k)}\left(T_{k}\right)^{-1} \gamma^{(k+1)}\left(T_{k+1}\right)\right) \\
& =\bar{\pi}_{k+1}\left(E_{k}\right)+\sum_{i=1}^{r}\left[-Z_{i}, \underline{\gamma}\left(b_{i}\right)-\underline{\gamma}\left(a_{i}\right)\right] \\
& =0 .
\end{aligned}
$$


This concludes the proof.

We now prove Theorem 1.2. The proof is basically the same as that of Theorem 4.1 and we just list the required minor modifications below.

Proof of Theorem 1.2. The constraints imposed on the curves $\gamma^{(k)}$, as well as the cut and correction operations, have to be replaced by their symmetric counterparts. For $k=1, \ldots, s$ we inductively construct horizontal curves $\gamma^{(k)}:\left[-T_{k}, T_{k}\right] \rightarrow G$ parametrized by arclength satisfying:

(i') $\gamma^{(k)}\left(-T_{k}\right)=\gamma(-T)$;

(ii') $\gamma(T)^{-1} \gamma^{(k)}\left(T_{k}\right) \in G_{k+1}$ (in particular, $\gamma^{(s)}\left(T_{s}\right)=\gamma(T)$ );

(iii') $L\left(\gamma^{(k)}\right)<L(\gamma)$, i.e., $T_{k}<T$;

(iv') $T_{k} \geq T_{k-1}$ if $k \geq 2$;

(v') $L\left(\gamma^{(k)}\right) \leq L(\gamma)-(1+o(1)) \eta^{1+\beta}$

(vi') $\left.\underline{\gamma}^{(k)}\right|_{\left[2 \eta^{\left.\varrho_{k}, T_{k}\right]}\right.}=\left.\underline{\gamma}\right|_{\left[2 \eta^{\left.\varrho_{k}+\left(T-T_{k}\right), T\right]}\right.}\left(\cdot+\left(T-T_{k}\right)\right)$ and

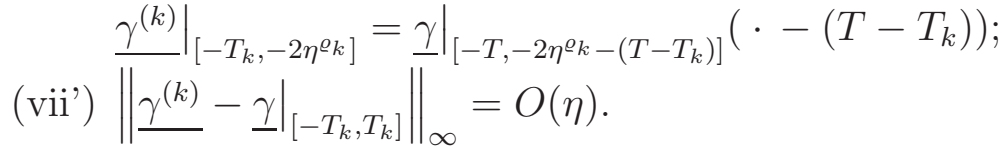

We list the necessary modifications in the various steps.

Step 1. The first curve is $\gamma^{(1)}:=\operatorname{Cut}^{\prime}(\gamma ;[-\eta, \eta])$, which satisfies (i')-(vii') for $k=1$.

Step 2. The interval $I_{k}$ is now $\left[-\eta^{\varrho_{k}}, \eta^{\varrho_{k}}\right]$.

Step 3. Let $E_{k}, \tau_{k}, g_{k}$ be defined as in the proof of Theorem 4.1. The estimate $\pi_{k+1}\left(g_{k}\right)=O\left(\eta^{(k+1) \varrho_{k}}\right)$ follows by applying (2.6) to the curve

$$
\left.\left.\gamma\left(\tau_{k}\right)^{-1} \gamma\right|_{\left[-\tau_{k}, \tau_{k}\right]}\left(\tau_{k}-\cdot\right) * \gamma^{(k)}\right|_{\left[-2 \eta^{\varrho_{k}, 2 \eta^{\varrho} k}\right]}
$$

and observing that $\gamma\left(-\tau_{k}\right)=\gamma^{(k)}\left(-2 \eta^{\varrho_{k}}\right)$. The length of the curve in (4.22) is $2 \tau_{k}+$ $4 \eta^{\varrho_{k}} \leq 10 \eta^{\varrho_{k}}$.

Step 4. In the definition (4.21) of $\gamma^{(k+1)}$, Cor is replaced by Cor'.

Step 5. The fact that $\gamma^{(k+1)}$ satisfies (vi') follows from the identity

$$
\underline{\gamma^{(k+1)}}=\left.\left.\left.\underline{\gamma^{(k)}}\right|_{\left[-T_{k},-\eta^{\varrho_{k+1}}\right]} * \underline{\gamma^{(k+1)}}\right|_{J_{k}} * \underline{\gamma^{(k)}}\right|_{\left[\eta^{\left.\varrho_{k+1}, T_{k}\right]}\right.}\left(\cdot+T_{k+1}-T_{k}\right),
$$

with $J_{K}:=\left[-\eta^{\varrho_{k+1}}-\left(T_{k+1}-T_{k}\right), \eta^{\varrho_{k+1}}+\left(T_{k+1}-T_{k}\right)\right]$, where the final point of each curve in the concatenation coincides with the starting point of the next one.

We finally prove Theorem 1.1 and then state its one-sided version.

Proof of Theorem 1.1. As mentioned in the introduction, it is not restrictive to assume that the Carnot-Carathéodory structure $(M, \mathscr{X})$ is that of a Carnot group $G$. To see this, consider the following facts:

(i) if $\gamma$ is length-minimizing in $(M, \mathscr{X})$ and $\kappa \in \operatorname{Tan}(\gamma ; t)$, then $\kappa$ is lengthminimizing in the nilpotent approximation $\left(M^{\infty}, \mathscr{X}^{\infty}\right)$, see [20, Theorem 3.6];

(ii) if $\kappa \in \operatorname{Tan}(\gamma ; t)$ and $\widehat{\kappa} \in \operatorname{Tan}(\kappa ; 0)$, then $\widehat{\kappa} \in \operatorname{Tan}(\gamma ; t)$, see [20, Proposition 3.7]. 
As a consequence, it suffices to show Theorem 1.1 for $\left(M^{\infty}, \mathscr{X}^{\infty}\right)$. Finally:

(iii) the nilpotent approximation $\left(M^{\infty}, \mathscr{X}^{\infty}\right)$ admits a Carnot group lifting $G$ with projection $\pi^{\infty}: G \rightarrow M^{\infty}$ (see [20, Definition 4.2]) and the following holds: if $\bar{\kappa}$ is a horizontal lift of $\kappa$ to $G$ (i.e., $\kappa=\pi^{\infty} \circ \bar{\kappa}$ ), then $\bar{\kappa}$ is length-minimizing in $G$ and $\kappa$ is a horizontal line in $M^{\infty}$ if $\bar{\kappa}$ is a horizontal line in $G$, see [20, Proposition 4.4];

(iv) the projection $\pi^{\infty}$ maps $\operatorname{Tan}(\bar{\kappa} ; 0)$ into $\operatorname{Tan}(\kappa ; 0)$, see [20, Proposition 4.3].

Hence, we are left to prove Theorem 1.1 for $G$. We can also assume that $t=0$ and $\gamma(0)=0$.

Let $\eta_{i} \downarrow 0$ be the sequence provided by Theorem 1.2 . Since $\operatorname{Exc}\left(\gamma ;\left[-\eta_{i}, \eta_{i}\right]\right) \rightarrow 0$, we can find a sequence $\zeta_{i} \downarrow 0$ satisfying

$$
\zeta_{i}^{-1 / 2} \operatorname{Exc}\left(\gamma ;\left[-\eta_{i}, \eta_{i}\right]\right) \rightarrow 0
$$

Let us set $\lambda_{i}:=\zeta_{i} \eta_{i} \downarrow 0$. Up to subsequences, using Lemma 2.6 and a diagonal argument, we can assume that there exists a length minimizer $\gamma_{\infty}: \mathbb{R} \rightarrow G$ parametrized by arclength such that

$$
\gamma_{i}(t):=\delta_{\lambda_{i}^{-1}}\left(\gamma\left(\lambda_{i} t\right)\right) \rightarrow \gamma_{\infty}(t)
$$

uniformly on compact subsets of $\mathbb{R}$, and that $\underline{\dot{\gamma}_{i}} \rightarrow \underline{\dot{\gamma}_{\infty}}$ in $L_{l o c}^{2}(\mathbb{R})$. For any fixed $N>0$ we have by Remark 2.4

$$
\operatorname{Exc}\left(\gamma_{i} ;[-N, N]\right)=\operatorname{Exc}\left(\gamma ;\left[-N \zeta_{i} \eta_{i}, N \zeta_{i} \eta_{i}\right]\right) \leq\left(N \zeta_{i}\right)^{-1 / 2} \operatorname{Exc}\left(\gamma ;\left[-\eta_{i}, \eta_{i}\right]\right) \rightarrow 0
$$

the last inequality being true for any $i$ such that $N \zeta_{i} \leq 1$. So, by Remark 2.5, we deduce that $\operatorname{Exc}\left(\gamma_{\infty} ;[-N, N]\right)=0$, which means that $\dot{\gamma}_{\infty}(t)$ is contained in a hyperplane $\mathfrak{h}_{1}$ of $\mathfrak{g}_{1}$ for a.e. $t \in[-N, N]$. Since this is true for any $N$, we deduce that there exists a hyperplane $\mathfrak{h}_{1}$ of $\mathfrak{g}_{1}$ such that $\underline{\dot{\gamma}}_{\infty}(t) \in \mathfrak{h}_{1}$ for a.e. $t \in \mathbb{R}$; in particular, $\gamma_{\infty}$ is contained in the Carnot subgroup $H$ associated with the Lie algebra generated by $\mathfrak{h}_{1}$.

If the rank of $G$ is $r=2$, we conclude that $\gamma_{\infty}$ is contained in a one-parameter subgroup of $G$. Since $\gamma_{\infty} \in \operatorname{Tan}(\gamma ; 0)$ is a length minimizer parametrized by arclength, we deduce that $\gamma_{\infty}$ is a line in $G$.

Otherwise, we can reason by induction on $r>2$ : since $H$ has rank $r-1$ and $\gamma_{\infty}$ is a length minimizer in $H$ parametrized by arclength, there exists $\widehat{\gamma} \in \operatorname{Tan}\left(\gamma_{\infty} ; 0\right)$ such that $\widehat{\gamma}$ is a line in $H \subset G$. By [20, Proposition 3.7] we have $\widehat{\gamma} \in \operatorname{Tan}(\gamma ; 0)$ and the proof is accomplished.

We state without proof the following version of Theorem 1.1, which holds for extremal points of length minimizers; we refer to [20, Section 3] for the definitions of the one-sided tangent cones $\operatorname{Tan}^{+}(\gamma ; 0)$ and $\operatorname{Tan}^{-}(\gamma ; T)$ of a horizontal curve 
$\gamma:[0, T] \rightarrow M$. The proof uses the same arguments as the previous one and can be easily deduced from Theorem 4.1 .

Theorem 4.2. Let $\gamma:[0, T] \rightarrow M$ be a length minimizer parametrized by arclength in a Carnot-Carathéodory space $(M, d)$. Then, each of the tangent cones $\operatorname{Tan}^{+}(\gamma ; 0)$ and $\operatorname{Tan}^{-}(\gamma ; T)$ contains a horizontal half-line.

Remark 4.3. In view of [20, Remark 3.10], Theorem 1.1] can be equivalently stated as follows: let $\gamma:[-T, T] \rightarrow M$ be a length minimizer in $(M, d)$ parametrized by arclength and let $h \in L^{\infty}(-T, T)$ denote the controls of $\gamma$; then, for any $t \in(-T, T)$, there exist an infinitesimal sequence $\eta_{i} \downarrow 0$ and a constant unit vector $v \in S^{r-1}$ such that

$$
h\left(t+\eta_{i} \cdot\right) \rightarrow v \quad \text { in } L_{l o c}^{2}(\mathbb{R}) .
$$

Of course, an analogous version holds for extremal points.

\section{REFERENCES}

[1] A. A. Agrachev, Some open problems. In Geometric Control Theory and Sub-Riemannian Geometry, Springer INdAM Series 5 (2014), 1-14.

[2] A. A. Agrachev \& Y. L. Sachkov, Control theory from the geometric viewpoint. Encyclopaedia of Mathematical Sciences, 87. Control Theory and Optimization, II. Springer-Verlag, Berlin (2004), xiv+412.

[3] W. K. Allard, On the first variation of a varifold. Ann. of Math. (2) 95 (1972), 417-491.

[4] A. Bellä̈che, The tangent space in subriemannian geometry. In Subriemannian Geometry, Progress in Mathematics 144, ed. by A. Bellaïche and J. Risler, Birkhäuser Verlag, Basel, 1996.

[5] L. C. Evans, Quasiconvexity and partial regularity in the calculus of variations. Archive for Rational Mechanics and Analysis 95 (1986), no. 3, 227-252.

[6] E. Giusti, Minimal surfaces and functions of bounded variation. Monographs in Mathematics, 80. Birkhäuser Verlag, Basel, 1984.

[7] E. Hakavuori \& E. Le Donne, Non-minimality of corners in subRiemannian geometry. Invent. Math. 206 (2016), no. 3, 693-704.

[8] H. Hermes, Nilpotent and High-Order Approximations of Vector Field Systems. SIAM Rev. 33 (1991), no. 2, 238-264.

[9] F. Jean, Control of Nonholonomic Systems: from Sub-Riemannian Geometry to Motion Planning. Springer Briefs in Mathematics. Springer, Cham, 2014.

[10] A. W. Knapp, Lie groups beyond an introduction. Progress in Mathematics, 140. Birkhäuser 2002.

[11] E. Le Donne, G. P. Leonardi, R. Monti \& D. Vittone, Extremal curves in nilpotent Lie groups. Geom. Funct. Anal. 23 (2013), 1371-1401.

[12] E. Le Donne, G. P. Leonardi, R. Monti \& D. Vittone, Extremal polynomials in stratified groups. Comm. Anal. Geom., to appear. ArXiv preprint 1307.5235.

[13] E. Le Donne, G. P. Leonardi, R. Monti \& D. Vittone, Corners in non-equiregular sub-Riemannian manifolds. ESAIM Control Optim. Calc. Var. 21 (2015), 625-634.

[14] G. P. Leonardi \& R. Monti, End-point equations and regularity of sub-Riemannian geodesics. Geom. Funct. Anal. 18 (2008), no. 2, 552-582. 
[15] F. MagGi, Sets of finite perimeter and geometric variational problems. An introduction to Geometric Measure Theory. Cambridge Studies in Advanced Mathematics, 135. Cambridge University Press, Cambridge, 2012.

[16] R. Montgomery, Abnormal Minimizers. SIAM J. Control Optim. 32 (1994), no. 6, 1605-1620.

[17] R. Montgomery, A tour of subriemannian geometries, their geodesics and applications. Mathematical Surveys and Monographs, 91. American Mathematical Society, Providence, RI, 2002. $\mathrm{xx}+259$.

[18] R. Monti, Regularity results for sub-Riemannian geodesics. Calc. Var. Partial Differential Equations 49 (2014), no. 1-2, 549-582.

[19] R. Monti, The regularity problem for sub-Riemannian geodesics. In Geometric Control Theory and Sub-Riemannian Geometry, Springer INdAM Series 5 (2014), 313-332.

[20] R. Monti, A. Pigati \& D. Vittone, On tangent cones to length minimizers in CarnotCarathéodory spaces. ArXiv preprint 1707.07990, 2017.

[21] L. Rifford, Singulières minimisantes en géometrié sous-riemannienne, Séminaire Bourbaki, Mars 2016 68ème année, 2015-2016, no. 1113.

[22] H. J. Sussmann, A regularity theorem for minimizers of real-analytic subriemannian metrics. Proceedings of the 53rd IEEE Conference on Decision and Control (2015), 4801-4806.

[23] D. Vittone, The regularity problem for sub-Riemannian geodesics. In Geometric Measure Theory and Real Analysis. Ed. by Luigi Ambrosio. Pisa: Scuola Normale Superiore, 2014, pp. 193-226.

E-mail address: monti@math.unipd.it

E-mail address: alessandro.pigati@math.ethz.ch

E-mail address: vittone@math.unipd.it

(Monti and Vittone) Università di Padova, Dipartimento di Matematica, via Trieste 63, 35121 PAdova, Italy

(Pigati) Scuola Normale Superiore, Piazza dei Cavalieri 7, 56126 Pisa, Italy

(Pigati) ETH Zürich, Department of Mathematics, RÄmistrasse 101, 8092 Zürich, SWITZERLAND 